\title{
Reactive power management to enhance solar energy penetration in small grids: technical and framework approaches
}

\author{
B. González-Díaz ${ }^{1}$, J.F. Gómez-González ${ }^{1}$, D. Cañadillas-Ramallo², J.A. Méndez-Pérez ${ }^{3}$ and R. \\ Guerrero-Lemus $^{2}$

\begin{abstract}
${ }^{1}$ Departamento de Ingeniería Industrial, Escuela Superior de Ingeniería y Tecnología, Universidad de La Laguna (ULL), Escuela Superior de Ingeniería y Tecnología, Universidad de La Laguna (ULL), Apdo. 456, 38206 La Laguna (Tenerife), España, phone: +34 922316502 EXT 6252, email: bgdiaz@ull.edu.es
\end{abstract} \\ ${ }^{2}$ Departamento de Física, Facultad de Ciencias, Universidad de La Laguna (ULL), \\ Apdo. 456, 38206 La Laguna (Tenerife), España.
}

${ }^{3}$ Departamento de Ingeniería Informática y de Sistemas, Escuela Superior de Ingeniería y Tecnología, Universidad de La Laguna (ULL), Apdo. 456, 38206 La Laguna (Tenerife), España.

\begin{abstract}
In the last few years, rising environmental concerns and especially, cost reductions that photovoltaic technology has experienced, have led to an unprecedented increase in the deployment of photovoltaic systems all over the world. Although nowadays most of the installed photovoltaic capacity is due to utility-scale plants, medium- and small-scale plants connected to distribution systems are becoming increasingly more frequent. Distribution networks were originally designed assuming a centralized operation, and the growing penetration of distributed generators is arising both technical and regulatory issues. In this work, we summarize different approaches from the technical and framework point of view.
\end{abstract}

\section{Key words}

Reactive power management, photovoltaic energy, control strategies.

\section{Introduction}

A small grid is defined as an isolated small-scale power network, designed for a low voltage distribution system to provide a power supply for a region or an island [1]. Nowadays, the small grids operate in two modes: (i) grid connected or (ii) individual/islanded mode, completely independent of the grid.

The incorporation of solar photovoltaic (PV) systems into electrical grids has extraordinarily increased in the recent years, mainly due to the attractive installation cost, driven policies and the relevance of the environmental issues in some countries. Annual new solar PV system installations increased from 29.5 GW in 2012 to $79.4 \mathrm{GW}$ in 2016, reaching a total cumulative grid connected PV installed capacity near to $440 \mathrm{GWp}$ [2], [3].
According to the Word Wind Energy Association [4], the overall capacity of all wind turbines installed worldwide by the end of 2017 reached near $540 \mathrm{GW}$.

The increasing penetration of Distributed Generation (DG), as the PV installations in the distribution systems, particularly in low voltage networks, has presented several important challenges (both technical and regulatory) over these systems. Distribution networks were originally designed and operated radially, based on the assumption of centralized generation in which power flows only occur in one direction, from the substation to the loads. However, the high deployment of DG in distribution grids has supposed a change in the original paradigm of electricity production, shifting from a centralized to a decentralized approach, generators of different sizes and multidirectional power flows.

\section{Technical approaches}

In the case of the small grids with an effective and relevant incorporation of $\mathrm{PV}$ and wind generators, storage devices, different loads, and power controllers, the connexion between the elements are carried out by means of power converters. In the management of a grid, the use of power converters offers a broad possibilities optimal operation and flexible control [5]. However, these power electronic devices arise new power quality problems in a microgrid, such as voltage harmonics, voltage sags, voltage swells, voltage unbalance, current harmonics, reactive power compensation, among other problems [6].

Therefore, reactive power dispatch and management acquire an important role in the power systems, operations, especially to manage voltage stability and line losses [7]. In distribution systems and microgrids, where the ratio of resistance to reactance is higher than in 
transmission systems, local reactive power management is a promising technique to reduce the power losses, and thereby the operational costs [8].

From the technical point of view, different analysis have been carried out in the literature, in order to stablish the optimal allocation and operation of reactive power compensation devices in distribution systems [6], [9].

Traditional devices to manage the power factor used in the distribution systems, as capacitor banks, among others [10], have been largely used in the electrical systems. However, the entrance of inverter-based distributed energy resources, as PV systems the use of these inverters for local reactive power compensation with faster response, managing the voltage regulation more accurately, especially during transient disturbances [11].

Despite of the studies about the centralized reactive power for Low Voltage (LV) grids [8], several authors have considered the local management of the reactive power by means of different methods. For example, using Optimal reactive power dispatch (ORPD) or even high voltage direct current (HVDC) transmission [12], the stochastic multi-objective optimal dispatch [13] or adaptive differential evolution algorithm [14], to enhance the PV penetration in the lines.

The incorporation of the wind power energy in the grids have been largely studied during the last decades. Different configurations of synchronous and asynchronous generators and their modifications have been largely used and studied in the literature. Depending on the generation technology, a large amount of solutions has been reported, such as Static Synchronous Compensators (STATCOMs) and Flexible AC Transmission system (FACTs) controllers [15], where the impact of STATCOM on grid integration of generation system has been investigated during normal condition and during faults. In these case, the results STATCOM has a great impact on the generator integration and stabilize the electrical grid by provide reactive power at the starting and during the fault.

Wind farms are usually considered as PV or PQ nodes for load flow or reactive power studies [16]. Usually, the stability of the voltage in electrical grids with wind generators is carried out assigning wind generation as PQ buses with $\cos$ phi $=0$. However, there is not any purpose to contribute to the voltage stability by using the DoublyFed Induction Generator (DFIG) as a reactive power generator. However, the reactive power capability of the wind induction machine can be used to minimize the power line losses in the reactive power dispatch, considering that the wind generators are already fixed at specific places in the network. In these approaches, the studies are not designed to find out the optimal places for locating wind power farms, in order to improve the voltage stability of the control area either. Moreover, the needed reactive power is supposed to be injected only by the induction electrical machine and not by the grid side converter.

In contrast to classical DFIG models reported in the literature, the authors in [16] proposed models are not constrained to the classic PQ or PV assignation. In this work, the authors consider the reactive power capability from the DFIG induction machine and from the grid side converter in the optimization process. This approach has the advantage of representing current DFIG operations more realistically.

In the case of photovoltaic systems, the use of intelligent inverters or with advanced functions in photovoltaic systems can be key to achieving a greater penetration of renewable energies within the electrical system. These investors are able to monitor the state of the network, respond autonomously to variations that may produce instabilities, maintain the quality of supply and even provide auxiliary services, such as reactive energy management. This last characteristic is fundamental, not only because the loads connected to the distribution networks require reactive energy, but also because it offers the possibility of guaranteeing that the voltage remains within legal requirements [17].

PV systems must inject electrical energy into the distribution network, either in low or medium voltage, so the voltage will necessarily rise at the evacuation point so that the energy reaches the consumption points. When the associated consumption is reduced near the PV installation, the increase in voltage must be even greater, which may cause the legal limits to be exceeded in certain situations. It is in these cases, reactive energy plays a key issue: consuming reactive energy (reactive inductive) reduces the voltage, while supplying reactive energy (reactive capacitive) increases the voltage level [18]. Therefore, an inverter capable of autonomously regulating the generation or consumption of reactive energy may also control the voltage and, thereby, ensure the quality of the supply. In this way, it is possible to increase the number of distributed generators that the network would allow, and that existing ones operate more hours since before reaching the upper limit of admissible voltage and disconnecting they will be able to continue delivering active to the network and reduce the voltage through the reactive

The functionality of these investors can be implemented through four different strategies [10]:

- Fixed value of reactive power (Q).

- Fixed value of the power factor $(\cos \varphi)$.

- Variation of the power factor according to the active energy injected $(\cos \varphi(\mathrm{P}))$.

- Variation of the reactive energy as a function of the local voltage of the network $(\mathrm{Q}(\mathrm{V}))$.

In response to the problems presented by distributed generation, the fourth option interesting. The inverter would operate in the following way: when a voltage value higher than the established limit is detected, the inverter will consume inductive reactive power to reduce the voltage at the point of connection to the network. On the other hand, when measuring a lower voltage value, the inverter will inject capacitive reactive power, thus increasing the voltage is increased until the desired level.

\section{Framework approaches}

Regardless the different technical approaches, a clear framework is necessary to stablish an adequate method to 
achieve an important PV penetration into the electrical grids. Germany has recently developed a grid code VDEAR-N 4105 that regulates the interconnection of DG in LV networks, allowing the reactive power. In USA, a revision for their IEEE 1547 standard has been recently published, providing more flexibility to the interconnection of PV generators allowing advanced functionalities of the inverters to provide voltage control at the distribution level.

Germany has developed the standard VDE-AR-N 4105: 2011-08 for the connection of generating systems in low voltage networks. In the USA, the IEEE 1547 standard is applied for the connection of distributed generation in electrical systems.

The VDE standard AR N 4105 establishes a series of restrictions that are summarized in Table I.

Table I. - Parameters established by the VDE standard AR N 4105: 2011

\begin{tabular}{|c|c|}
\hline \multicolumn{2}{|c|}{$\begin{array}{l}\text { System parameters for application of the VDE } \\
\text { standard AR N 4105: } 2011\end{array}$} \\
\hline Application voltage & $\leq 1 \mathrm{kV}$ \\
\hline Application power & $<100 \mathrm{kVA}$ \\
\hline \multicolumn{2}{|c|}{$\begin{array}{c}\text { Reactive compensation } \\
(\text { Only if: } \mathrm{P}>20 \%,-10 \%<\mathrm{V}<+10 \%)\end{array}$} \\
\hline Smax $<=3,68 \mathrm{kVA}$ & 0,95 ind. - 0,95 cap. \\
\hline $\begin{array}{l}3,68 \mathrm{kVA}<\mathrm{Smax}<= \\
13,8 \mathrm{kVA}\end{array}$ & 0,95 ind. - 0,95 cap. \\
\hline Smax $>13,8 \mathrm{kVA}$ & 0,90 ind. - 0,90 cap. \\
\hline Frequency range & $\begin{array}{l}47,5-51,5 \mathrm{~Hz} \\
(-5 \%-+3 \%)\end{array}$ \\
\hline \multicolumn{2}{|c|}{ Reconnection } \\
\hline Voltage & $85 \%-110 \%$ \\
\hline Frequency & $47,5-50,05 \mathrm{~Hz}$ \\
\hline
\end{tabular}

The reactive energy production of an inverter is limited by the active and apparent energy. Therefore, when the maximum production of active energy is generated, the capacity of the reactive compensation is reduced. In addition, the inverters power in the market are staggered and therefore, the power available is discrete. In some cases, the required active power can be higher than the peak power of the photovoltaic plant. Therefore, a small account of available energy is required to perform this regulation.

\section{Analysis}

To analyse the reactive power management strategy with smart inverters, a PV power plant behaviour in a Low Voltage grid placed at the end of the line have been simulated. The total power of the PV plant placed at the end of the line is the $45 \mathrm{~kW}$ [19].

The analysis performed in the present work in response to the problem of overvoltage, so the results are presented in the form of a voltage profile. The voltage profile along the line is the representation of the voltage level as a function of the distance to the transformation centre. As indicated above, in the case of overvoltage, the most unfavourable situations occur when there is large production and low consumption in the line.
The line has been modelled considering the maximum PV production and low demand for the loads for each case. Regarding to voltage control, two of the strategies included in the German standard normative have been simulated:

A. Control of the power factor as a function of the active power, $\cos \varphi(P)$.

This method calculates the reactive energy as a function of the active power produced, by varying the power factor. This mode of operation does not require the inverter to have a voltage measurement system on its output to the distribution network. However, due to this lack of information about the grid voltage, in many cases this strategy force to the reactive power even when is not necessary to preserve the voltage level within the limits. Also in this strategy, the inverters are active, independently of their position in the network, near the transformation centre or at the end of the line.

According to the German standard, for installations with apparent power lower than $13.8 \mathrm{kVA}$, the regulation of $\cos \varphi$ will be between 0.95 and 1 ; while for inverters with higher powers it will be between 0.9 and 1 .

B. Control of reactive energy as a function of voltage, $Q$ $(V)$. This method does use the inverter's connection point information to calculate reactive energy needs (both consumption and production). For the implementation of this control strategy, the reactive energy characteristic curve has been used as a function of the voltage. The inverters begin to consume / supply reactive energy when the value of the voltage deviates $4 \%$ of the nominal value [10]. This type of strategy can be implemented in two ways:

Local control: In this method, each inverter is working according to the voltage level in the connection circuit. For this reason, the inverters closest to the transformation centre, whose voltage values not strongly perturbed by the PV installation, will not participate actively in the voltage regulation. Consequently, the inverters located at the end of the line will be operating at maximum capacity for longer time.

Global or intelligent control: In this method, the inverters communicate with a control station, which optimizes the management of the reactive energy in order to do not exceed the voltage limits in any section of the line. Although this control requires communication infrastructures between the inverters, to optimize reactive energy flows and maintain the voltage profiles inside of the limits.

In the figure 1 the voltage profiles resulting from the simulations carried at the initial stage with a $45 \mathrm{~kW} \mathrm{PV}$ power plant without $\mathrm{Q}(\mathrm{V})$ or $\cos \varphi(\mathrm{P})$ control strategies are shown. 


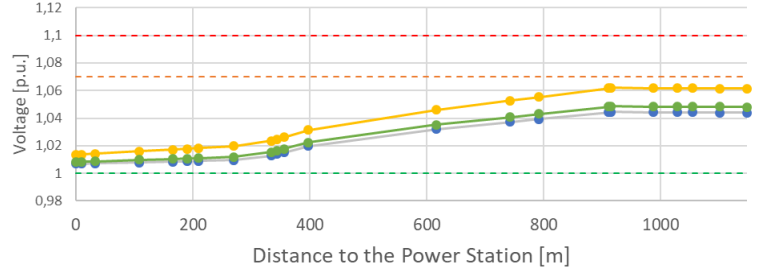

Fig. 1. Initial voltage profiles: no control strategy (yellow), Q(U) (blue) and $\cos \varphi$ (green).

The implementation of control strategies modified the voltage profile, decreasing the values along the line to accomplish the legal limits. Also, the Q (V) control strategy is the most effective to reduce the overvoltage. These results are obtained inside to an operation range between 0.8 and 1, while the control of $\cos \varphi(\mathrm{P})$ implemented (extracted from the German standard) only regulates between 0.9 and 1 , for what is less adequate to consume / produce reactive energy [10].

The effect of the incorporation of another photovoltaic plant at the end of the line has been considered. Through an iterative process of energy flows considering the control strategies, the maximum photovoltaic power allowed at the end of the line, preserving the voltage under the upper limit, has been only $3.9 \mathrm{~kW}$. However, using control strategies, a capacity of $19 \mathrm{~kW}$. can be achieved using the control Q (V). Moreover, an installation of $12 \mathrm{~kW}$ using the $\cos \varphi(\mathrm{P})$ control can be allocated.

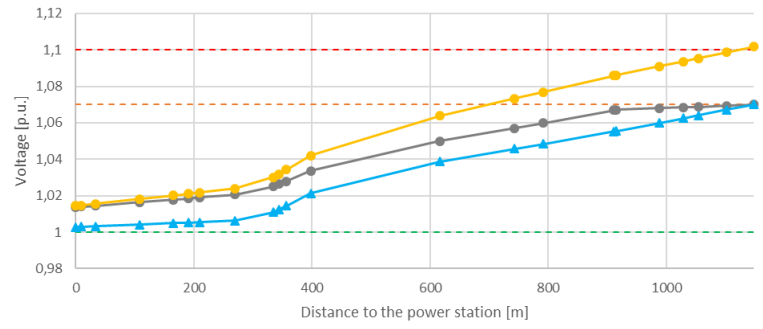

Fig. 2. Voltage profiles of a new PV installation: Maximum power for PV installation available, $3.9 \mathrm{~kW}$ (grey), increment of the $19 \mathrm{~kW}$ on the $\mathrm{PV}$ power using $\mathrm{Q}(\mathrm{U})$ control (blue) and without control strategy (yellow).

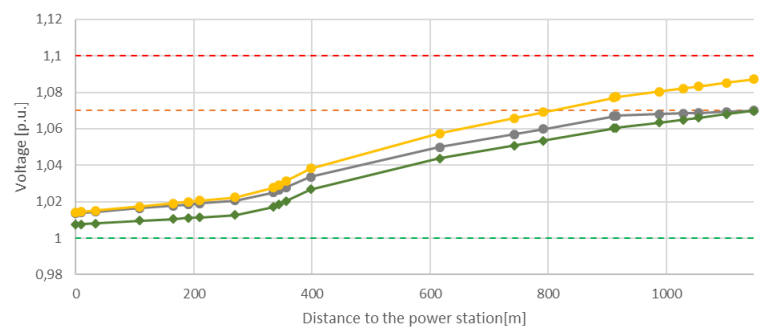

Fig. 3. Voltage profiles of a new PV installation: Maximum power for PV installation available, $3.9 \mathrm{~kW}$ (grey), increment of the $12 \mathrm{~kW}$ on the PV power using $\cos \varphi(\mathrm{P})$ control (green) and without control strategy (yellow).

As it is shown in the figures 2 and 3, the control thru $\cos \varphi$ (P) strategy allows lower PV power allocation in comparison with the $\mathrm{Q}(\mathrm{V})$ control. This fact is due to a higher operating range in the case of the control $Q(V)$ with respect to $\cos \varphi(\mathrm{P})$.
For the last scenario, six PV power plants have been distributed uniformly along the low voltage electric line. By means of the same iterative process of power flows calculation, used in the previous section, the additional maximum PV power capacity that can be managed in the line is $10.2 \mathrm{~kW}$ (the PV power of each installation is 1.7 $\mathrm{kW}$ ), without any control system.

In the case when the local Q (V) control is applied, in addition of the $45 \mathrm{~kW}$ previously installed, the total PV power in the line added is $57 \mathrm{~kW}(11 \mathrm{~kW}$ maximum per plant). In the study case when the $\mathrm{Q}(\mathrm{V})$ control is performed, an increment of $66 \mathrm{~kW}$ of PV energy can be managed in the electric line $(13.2 \mathrm{~kW}$ per $\mathrm{PV}$ power plant). Finally, using the $\cos \varphi(\mathrm{P})$ regulation, the total $\mathrm{PV}$ power added into the line is $30 \mathrm{~kW}(5 \mathrm{~kW}$ power in each PV power plant).

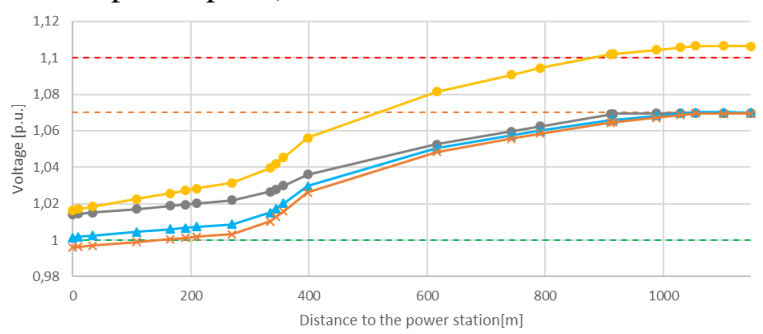

Fig. 4. Voltage profile using $\mathrm{Q}(\mathrm{V})$ control local (blue), Q(V) control global (orange) in comparison with an increment of the same power, $9.5 \mathrm{~kW}$ per PV power plant without control (yellow) and the real capacity of the increment of the line, $1.7 \mathrm{~kW}$ per PV power plant (grey).

In the figure 4 it is shown the voltage profiles obtained after applying the $\mathrm{Q}(\mathrm{V})$ regulation, both local and global. According to the figure 3 with global control Q (V) a few more power can be injected in comparison with the local strategy. The origin of this difference underlies in the fact that in local control, the inverters closest to the transformation centre are not contributing to the control management, because the voltage level in their connection points are inside of the regulation limits. In the case of the global regulation, all inverters are contributing to the regulation, allowing deeper penetration of photovoltaics in the line.

Finally, the $\cos \varphi(\mathrm{P})$ control was modelled for distributed plants. The theoretical maximum capacity with this control strategy is $5 \mathrm{~kW}$ per plant, being able to manage a total amount of $30 \mathrm{~kW}$ in total.

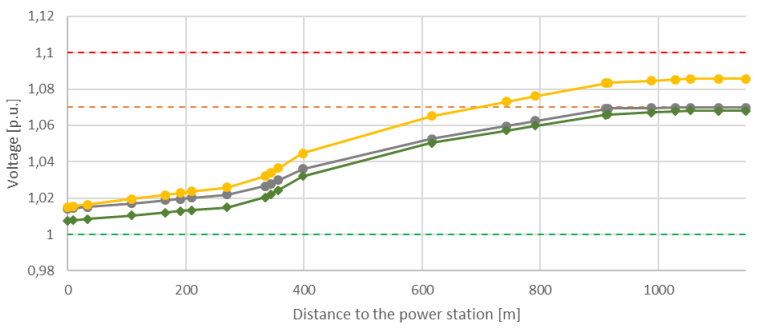

Fig. 5. Voltage profile using $\cos \varphi(\mathrm{P})$ control (green) in comparison with an increment of the same power, $5 \mathrm{~kW}$ per PV power plant without control (yellow) and the real capacity of the increment of the line, $1.7 \mathrm{~kW}$ per PV power plant (grey). 
An analysis of the studied scenarios reveals that the differences in the voltage at the beginning of the line are mainly due to the injected reactive energy by the inverters, since all the scenarios were simulated with the same tap capacity of the transformer of the transformation centre.

The operational range of both control strategies are different. The control windows of $\mathrm{Q}(\mathrm{V})$ control reveals a major range, being able to achieve values from $\cos \varphi=1$ to reach $\cos \varphi=0.8$. In the $\cos \varphi(\mathrm{P})$ control strategy, a maximum value of $\cos \varphi=0.9-0.95$ can be reached. In other words, this effect is mainly due to the advantage in the $\mathrm{Q}(\mathrm{V})$ strategy in comparison with the $\cos \varphi(\mathrm{P})$ strategy, according to the limits stabilised in German standard.

A strategy including a voltage regulation in the transformation centre can increase the total amount of the PV power. However, in the cases that the same transformer supplies several LV lines, the total PV power capacity in each line should be calculated taking into account the whole lines.

The voltage level of the line can be influenced by the presence of other equipment that supplies reactive power to the network, such as capacitor banks. Although in reality the consumption associated with the $45 \mathrm{~kW}$ plant has a battery of capacitors, in the models it has been chosen to isolate this effect so that the study is more significant in relation to photovoltaic systems.

The most modern inverters have the capacity to regulate the power factor between 0 and 1, both with capacitive and inductive behaviour ( $\cos \varphi=0-1 \mathrm{cap} /$ ind). However, the functionality of varying the $\cos \varphi$ in that range depends on the active power that is being injected by the inverter at each moment. New strategies are considering this technology, where the power factor can vary between 0.9 capacitive and 0.9 inductive. If the active power drops to $50 \%$ of the maximum power, the inverter will be able to operate with power factors between 0.45 capacitive and 0.45 inductive. In the extreme case that there is no active power injection, the inverters consume / produce reactive energy $(\cos \varphi=0$ inductive $/ \cos \varphi 0=$ capacitive respectively).

Although when the injection of active power is low, the probability of overvoltages in the line is lower, this functionality gives solar inverters the potential to become voltage regulators / reactive power of the network. Some inverter manufacturers are already developing concepts such as "Q on demand 24/7", which will allow investors to operate as regulators even at night. Furthermore, if this wide operational window were combined with the $\mathrm{Q}(\mathrm{V})$ and $\cos \varphi(\mathrm{P})$ controls simultaneously, the possibilities of voltage regulation, as well as the amount of photovoltaic power that a line could accommodate, would increase considerably.

\section{Conclusions}

In this work, the actual technical and normative approaches have been analysed.

Voltage regulation through the advanced functions of the inverters (reactive power control Q (V) or power factor cos $\varphi(\mathrm{P}))$ is shown as a fairly effective measure to mitigate the problem of overvoltages caused by the installation of photovoltaic plants in low voltage networks.

Using control mechanisms, such as the control mechanism studied in this work, the overvoltages registered along the line are reduced due to the PV power injection in the LV line.

The control of reactive energy as a function of voltage, Q $(\mathrm{V})$, is more effective, mainly because this method requires the input of the voltage levels and can perform the control loop according to the real values.

The control of the power factor according to the active power, $\cos \varphi(\mathrm{P})$, uses only the information of the active power supplied by the PV power plant and therefore, the optimum control cannot be performed successfully. This method is more accurate in the case of the installed inverters have not the capacity to measure the voltage levels in the LV line.

Applying the $\cos \varphi(\mathrm{P})$ control, the capacity of a LV line is adequate to increase a $17 \%$ (end of line) and 36\% (uniformly distributed along the line) PV power.

Applying the local Q (V) control, the total PV power added in the LV line reaches $31 \%$ (photovoltaic at the end of the line) and $85 \%$ (photovoltaic distributed uniformly in the line), in comparison with a system without any control strategy in the PV inverters.

Applying a Q (V) global control, the PV power that can be managed in the line increases between $31 \%$ (end of line) and $101 \%$ (uniformly distributed), in comparison with a system without any control strategy in the PV inverters.

The results show that the control strategy $\mathrm{Q}(\mathrm{V})$ is more effective to the control strategy $\cos \varphi(\mathrm{P})$ in order to increase the PV power penetration.

Moreover, the global Q (V) control is effective when the $\mathrm{PV}$ power is concentrated at the end of the line. However, this strategy requires communication infrastructures between the devices and the associated cost of this infrastructure lack its implementation. The results between a local or global control are strongly related to factors as the number of facilities to be regulated, the importance of the regulation, as well as the specific characteristics of each line.

\section{References}

[1] M. T. L. Gayatri, A. M. Parimi, and A. V. Pavan Kumar, "A review of reactive power compensation techniques in microgrids," Renew. Sustain. Energy Rev., vol. 81, no. August 2017, pp. 1030-1036, 2018.

[2] A. Jäger-Waldau, "Snapshot of photovoltaics-March 2017," Sustain., vol. 9, no. 5, pp. 1-9, 2017.

[3] A. Jaeger-Valdau, PV Status Report 2017, no. November. 2017.

[4] WWEA, "Word Wind Energy Association," 2018. [Online]. Available: http://wwindea.org/blog/2018/02/12/2017-statistics/. [Accessed: 15-Jan-2019].

[5] S. Bielecki and T. Skoczkowski, "An enhanced concept of Q-power management," Energy, vol. 162, pp. 335-353, 2018.

[6] R. Passey, T. Spooner, I. MacGill, M. Watt, and K. Syngellakis, "The potential impacts of grid-connected distributed generation and how to address them: A review of technical and non-technical factors," Energy 
Policy, vol. 39, no. 10, pp. 6280-6290, 2011.

[7] O. Gandhi, C. D. Rodríguez-Gallegos, W. Zhang, D. Srinivasan, and T. Reindl, "Economic and technical analysis of reactive power provision from distributed energy resources in microgrids," Appl. Energy, vol. 210, no. August 2017, pp. 827-841, 2018.

[8] S. X. Chen, Y. S. F. Eddy, H. B. Gooi, M. Q. Wang, and S. F. Lu, "A centralized reactive power compensation system for LV distribution networks," IEEE Trans. Power Syst., vol. 30, no. 1, pp. 274-284, 2015.

[9] J. Zhao, Z. Zhang, J. Yao, S. Yang, and K. Wang, “A distributed optimal reactive power flow for global transmission and distribution network," Int. J. Electr. Power Energy Syst., vol. 104, no. February 2018, pp. 524-536, 2019.

[10] J. F. Gómez-González et al., "Reactive power management in photovoltaic installations connected to low-voltage grids to avoid active power curtailment .," in International Conference on Renewable Energies and Power Quality (ICREPQ'18), 2018, no. 16.

[11] R. G. Wandhare and V. Agarwal, "Reactive power capacity enhancement of a PV-grid system to increase PV penetration level in smart grid scenario," IEEE Trans. Smart Grid, vol. 5, no. 4, pp. 1845-1854, 2014.

[12] S. Sayah, "Modified differential evolution approach for practical optimal reactive power dispatch of hybrid ACDC power systems," Appl. Soft Comput. J., vol. 73, pp. 591-606, 2018.

[13] M. Ghaljehei, Z. Soltani, J. Lin, G. B. Gharehpetian, and M. A. Golkar, "Stochastic multi-objective optimal energy and reactive power dispatch considering cost, loading margin and coordinated reactive power reserve management," Electr. Power Syst. Res., vol. 166, no. October 2018, pp. 163-177, 2019.

[14] W. S. Sakr, R. A. EL-Sehiemy, and A. M. Azmy, "Adaptive differential evolution algorithm for efficient reactive power management," Appl. Soft Comput. J., vol. 53, pp. 336-351, 2017.

[15] S. Ahsan and A. S. Siddiqui, "Dynamic compensation of real and reactive power in wind farms using

STATCOM," Perspect. Sci., vol. 8, pp. 519-521, 2016.

[16] H. Amaris and M. Alonso, "Coordinated reactive power management in power networks with wind turbines and FACTS devices," Energy Convers. Manag., vol. 52, no. 7, pp. 2575-2586, 2011.

[17] National Renewable Energy Laboratory (NREL), "Advanced inverter functions to support high levels of distributed solar," 2014. [Online]. Available: https://www.nrel.gov/docs/fy15osti/62612.pdf. [Accessed: 15-Jan-2019].

[18] L. Collins and J. K. Ward, "Real and reactive power control of distributed PV inverters for overvoltage prevention and increased renewable generation hosting capacity," Renew. Energy, vol. 81, pp. 464-471, 2015.

[19] D. Cañadillas-Ramallo et al., "Voltage profile analysis and performance of a photovoltaic power plant in a small low voltage distribution grid in Tenerife Island," Electr. Eng. Electron. J., vol. 1, no. 3, pp. 1-2, 2016. 